\title{
a Red Herring?
}

Several years after the first description of the antiphospholipid syndrome (APS) in $1983^{1}$, Nigel Harris warned that, although they do exist, patients with APS are as rare as black swans - and predicted that "the value of studying antiphospholipid antibodies (aPL) [might] easily be lost in a sea of overinterpreted and over-reported laboratory and clinical findings ${ }^{2}$." Twenty-seven years later, the Obstetric Task Force of the 14th International Congress on Antiphospholipid Antibodies has confirmed the continuing lack of evidence supporting an association between aPL and recurrent early miscarriage (REM $)^{3}$. They noted the absence of consistent, predictable clinical outcomes from therapeutic trials and suggested potential causes. Despite apparent adherence to the 1999 initial Sapporo ${ }^{4}$ and 2006 revised Sydney ${ }^{5}$ criteria, studies have used heterogeneous patient selection protocols, variable laboratory inclusion criteria, and small sample sizes. In addition, there has been a lack of pathological or genetic evaluation to determine the nature of pregnancy losses when they occurred. The Task Force expressed concern regarding what they termed the "considerable enthusiasm" for "diagnosing APS and treating as yet unrecognized obstetric morbidities in the setting of positive aPL results." Given the absence of critical studies of association and therapeutic benefit, they felt that such diagnostic fervor would serve only to further obscure the search for genuine associations and proven treatments.

After 30 years of APS investigations, it would be reasonable to expect increased understanding of the obstetric complications related to aPL, but unfortunately, despite or perhaps because of the "considerable enthusiasm" noted above, there is less clarity, particularly with regard to how or even whether REM fits into the puzzle $3,6,7,8$. At the Treatment and Evaluation of Recurrent Miscarriage (TERM) program in Toronto, we have been involved in therapeutic and observational studies involving aPL and REM over the same 30-year period.
The early ASA/P study [prednisone and acetylsalicylic acid (ASA) vs placebo (1988-94)] included patients with, among other autoantibodies, variable levels of anticardiolipin antibodies (aCL) and the lupus anticoagulant (LAC), which would not have met current APS criteria ${ }^{9}$. Likewise, the Hep/ASA study [low molecular weight heparin (LMWH) and ASA vs ASA alone, 2000-2004 ${ }^{10}$ also included patients with autoantibodies other than aPL, although we did include a subgroup analysis for that category (fulfilling Sapporo criteria). We also included patients with $\geq 2$ early losses, as well as $\geq 3$, which engendered considerable criticism at the time we reported our results ${ }^{11}$. Our practical rationale for our inclusion criteria was 3-fold: (1) it was becoming apparent that there was no etiological difference between women with 2 versus 3 early losses ${ }^{12,13}$; (2) if aPL were associated with REM, then the presence of the antibody, and not the number of losses, should be the determinant; and finally (3) we expanded the inclusion criteria to address extremely slow patient accrual, a universal issue in investigation of women with aPL and pregnancy morbidity, as evidenced by the similar small sample sizes in other therapeutic trials of this population ${ }^{3,10,14}$.

Our observational studies over the decades have all added evidence to support the following conclusions regarding aPL and REM: (1) patients with persistent moderate to high levels of aPL are rarely seen: we found $2.7 \%$ of 2257 patients with persistent confirmed LAC over 6 years ${ }^{15}$; Wahl, et al found $0.8 \%$ of 6321 patients with $\geq 2$ moderate to high aCL IgG or IgM over about 4 years ${ }^{16}$; and Pengo, et al found confirmed triple positive aPL in $3.6 \%$ of 1520 patients over 4 years ${ }^{17}$; (2) the vast majority of women with REM have low levels or no aCL or $\mathrm{LAC}^{15,17,18,19}$; (3) regardless of aPL positivity, women with REM have a good prognosis for subsequent live birth, independent of treatment with ASA alone, LMWH/ASA, or unfractionated heparin/ASA $3,10,14,19$.

See Prevalence and significance of aPL, page 210, and

Obstetric APS: lobsters only? Or should we also look for selected red herrings?, page 158

Personal non-commercial use only. The Journal of Rheumatology Copyright (c) 2015. All rights reserved. 
Our center is one of several across North America that has participated in the PROMISSE study (Predictors of pRegnancy Outcome: bioMarkers In antiphospholipid antibody Syndrome and Systemic lupus Erythematosus), a prospective, observational investigation of aPL and pregnancy outcome (regardless of the presence or absence of clinical criteria of APS, but excluding pregnancies that end before the first trimester). Interim analysis of 144 aPL-positive pregnancies found that the absence of LAC appeared to be a strong predictor of uncomplicated pregnancies in women, regardless of the presence of aCL or anti- $\beta 2$-glycoprotein I antibody ${ }^{20}$. We have also noted an association between persistent LAC and sporadic stillbirth $^{15}$, intrauterine growth restriction and HELLP (hemolysis, elevated liver enzymes and low platelet count), and persistent aPL with preterm delivery ${ }^{21}$. It is informative that although PROMISSE is a longterm multicenter international study that includes only stringently evaluated, high-risk patients who fulfill (core-laboratory determined) APS laboratory criteria, there were only 144 aPL-positive pregnancies with $14(9.7 \%)$ late losses between 2003 and 2011 This low rate of late obstetric mortality in a high-risk population highlights the rarity and complexity of this clinical entity and the difficulties in acquiring an accurately identified sample sufficiently large to study ${ }^{14,20,22}$.

If we take a moment to broaden rather than narrow our focus after 30 years' collective experience, we can see that "Obstetric APS" appears to have 2 general presentations: the most common includes women with low levels of aCL (but not LAC) and a history of exclusively early losses but no thrombosis (in the absence of any concomitant risk factors such as surgery, oral contraception, or the postpartum period). These patients have a high likelihood of live birth in a pregnancy subsequent to presentation regardless of therapeutic protocol, negligible risk of postpartum thrombosis, and a good longterm prognosis ${ }^{22}$. The less frequent presentation involves women with persistently high levels of aPL including LAC, a history of second or third trimester loss(es), and possibly a history of thrombotic events. These patients often have an unpredictable pregnancy course regardless of therapeutic protocol. Their longterm prognosis has been reported to include a potentially higher risk of thrombotic events despite continuous anticoagulation ${ }^{3,22,23}$.

There are no data in the literature regarding remission rates for APS. Cervera, et al recently reported $85 \%$ of 1000 patients had no thrombotic events over a 10-year observation period ${ }^{23}$. During the second 5-year followup period, $24.8 \%$ of patients taking antithrombotic treatment versus $5.2 \%$ of patients without any antithrombotic treatment developed thrombosis, and $8.1 \%$ versus $1.5 \%$, respectively, developed APS-related obstetric complications. Of the 135 patients who received no antithrombotic treatment, 93.3\% and $97.0 \%$, respectively, had no thrombotic or adverse obstetric events. In addition, investigators were unable to identify any clinical or laboratory prognostic factors for this syndrome and neither individual aPL nor any combination of them was associated with an increased incidence of any specific clinical manifestation. While a confirmed moderate to high aPL was an inclusion criterion for this cohort, there was no mention of whether aPL levels were measured or varied throughout the 10-year followup.

The current classification of APS is a permanent designation. The immutability of this label should therefore necessitate compelling laboratory and clinical evidence before it is applied. The expert panel that recommended revisions to the Sapporo criteria recognized this when they called for an extension of the laboratory criteria for repeated aPL measurements from not less than 6 to not less than 12 weeks apart ${ }^{3}$. This revision was intended to eliminate the risk of transient, infection-related aCL and artificially prolonged LAC due to anticoagulation following an acute thrombotic event. Perhaps even this period of aPL positivity is still too brief.

It is our contention that the current classification thresholds are too broad, and that patients with lower levels of aPL, no LAC, and REM only should not be given the APS designation at all. Only those patients who have a history of thrombotic events and/or late obstetric morbidity with consistently high aPL with or without a LAC should be classified as APS. This would eliminate the somewhat specious designations "obstetric APS" and "thrombotic APS" altogether and clarify the syndrome as simply "APS."

Many APS-related articles over the last 30 years have been inconclusive, ending with the authors calling for more multicenter studies to confirm their results ${ }^{1}$. As it currently stands, the APS classification system functions "like a fishing trawler [capturing] many more innocent subjects than it should" 24 . The resultant overdiagnosis caused by inclusion of women with REM and low or transient levels of aPL is hampering our understanding of this syndrome. We suggest that useful and conclusive data already exist in our collective studies, particularly with regard to therapeutic approaches to aPL-associated pregnancy, but it will be necessary to separate the intended catch from the bycatch.

We propose a collegial challenge: a collective, multicenter, retrospective analysis of results from all pregnancy-related observational and therapeutic trials ${ }^{3}$. Subgroup analysis should be restricted to include only patients with a history of late adverse obstetric events (with or without thrombosis) and persistently high levels of aPL. Collated results would add to those recently reported by Ruffatti, et al and would provide aPL-specific, treatment-associated pregnancy outcome data that refute, support, or advance the current standard of care ${ }^{25}$. Regardless of the findings, this proposed retrospective collaborative study would clarify and inform clinical decision making for physicians with pregnant patients and clearly defined APS. 
CHRISTINE A. CLARK, PhD,

Research Associate;

KAREN A. SPITZER, MSc,

Study Coordinator,

LifeQuest Centre for Reproductive Medicine

and Mt. Sinai Hospital;

CARL A. LASKIN, MD, FRCPC

Associate Professor,

Departments of Medicine, Obstetrics and

Gynecology and Immunology,

Faculty of Medicine, University of Toronto; and LifeQuest Centre for Reproductive Medicine, Toronto, Ontario, Canada.

Address correspondence to Dr. C.A. Clark, Suite 1800, 655 Bay St., Toronto, Ontario, Canada M5G2K4; E-mail: cclarksolo@aol.com

\section{REFERENCES}

1. Hughes GR. Thrombosis, abortion, cerebral disease, and the lupus anticoagulant. BMJ 1983;287:1088-9.

2. Harris EN. Syndrome of the black swan. Br J Rheumatol 1987;26:324-6.

3. de Jesus GR, Agmon-Levin N, Andrade CA, Andreoli L, Chighizola CB, Flint Porter T, et al. 14th International Congress on Antiphospholipid Antibodies Task Force report on obstetric antiphospholipid syndrome. Autoimmun Rev 2014;13:795-813.

4. Wilson WA, Gharavi AE, Koike T, Lockshin MD, Branch DW, Piette JC, et al. International consensus statement of preliminary classification criteria for definite antiphospholipid syndrome: report of an international workshop. Arthritis Rheum 1999;42:1309-11.

5. Miyakis S, Lockshin MD, Atsumi T, Branch DW, Brey RL, Cervera $\mathrm{R}$, et al. International consensus statement on an update of the classification criteria for definite antiphospholipid syndrome (APS). J Thromb Haemost 2006;4:295-306.

6. Branch DW, Silver RM, Porter TF. Obstetric antiphospholipid syndrome: current uncertainties should guide our way. Lupus 2010;19:446-52

7. Bouvier S, Cochery-Nouvellon E, Lavigne-Lissalde G, Mercier E, Marchetti T, Balducchi JP, et al. Comparative incidence of pregnancy outcomes in treated obstetric antiphospholipid syndrome: the NOH-APS observational study. Blood 2014;123:404-13.

8. Kwak-Kim J, Agcaoili MS, Aleta L, Liao A, Ota K, Dambaeva S, et al. Management of women with recurrent pregnancy losses and antiphospholipid syndrome. Am J Reprod 2013:69:596-607.

9. Laskin CA, Bombardier C, Hannah ME, Mandel FP, Ritchie JW, Farewell V, et al. Prednisone and aspirin in women with autoantibodies and unexplained recurrent fetal loss. N Engl J Med 1997;337:148-53.

10. Laskin CA, Spitzer KA, Clark CA, Crowther MR, Ginsberg JS, Hawker GA, et al. Low molecular weight heparin and aspirin for recurrent pregnancy loss: results from the randomized controlled Hep/ASA trial. J Rheumatol 2009;36:279-87.
11. Carp HJ. Low molecular weight heparin and aspirin for recurrent pregnancy loss: results from the HepASA trial [letter; author reply]. J Rheumatol 2010;37:202-3.

12. American College of Obstetricians and Gynecologists. Management of recurrent early pregnancy loss. ACOG Practice Bulletin No. 24. 2001;24:1-8.

13. Jaslow CR, Carney JL, Kutteh WH. Diagnostic features identified in 1020 women with 2 versus three or more recurrent pregnancy losses. Fertil Steril 2010;93:1234-43.

14. Clark CA, Laskin CA, Spitzer KA. Anticardiolipin antibodies and recurrent early pregnancy loss: a century of equivocal evidence. Hum Reprod Update 2012;18:474-84.

15. Clark CA, Davidovitz J, Spitzer KA, Laskin CA. The lupus anticoagulant: results from 2257 patients attending a high-risk pregnancy clinic. Blood 2013;122:341-7.

16. Wahl D, Devignes J, Mohamed S, Zuily S, Lecompte T. Definition and significance of high positivity aCL ELISA. Lupus 2012;21:725-6.

17. Pengo V, Ruffatti A, Del Ross T, Tonello M, Cuffaro S, Hoxha A, et al. Confirmation of initial antiphospholipid antibody positivity depends on the antiphospholipid antibody profile. J Thrombosis Haemost 2013;11:1527-31.

18. Clark CA, Spitzer KA, Nadler JN, Laskin CA. Low aCL IgG titres in recurrent pregnancy loss signal the need for redefining APS [abstract]. Arthritis Rheum 2002;46 Suppl:S50.

19. Cohn DM, Goddijn M, Middledorp S, Korevaar JC, Dawood F, Farquharson RG. Recurrent miscarriage and antiphospholipid antibodies: prognosis of subsequent pregnancy. $\mathrm{J}$ Thrombo Haemost 2010;8:2208-13.

20. Lockshin MD, Kim M, Laskin CA, Guerra M, Branch DW, Merrill $\mathrm{J}$, et al. Prediction of adverse pregnancy outcome by the presence of lupus anticoagulant, but not anticardiolipin antibody, in patients with antiphospholipid antibodies. Arthritis Rheum 2012;64:2311-8

21. Clark CA, Spitzer KA, Crowther MA, Nadler JN, Laskin MD, Waks JA, et al. Incidence of postpartum thrombosis and preterm delivery in women with antiphospholipid antibodies and recurrent pregnancy loss. J Rheumatol 2007;34:992-6.

22. Sciascia S, Cosseddu D, Montaruli B, Kuzendo A, Bertero MT. Risk scale for the diagnosis of antiphospholipid syndrome. Ann Rheum Dis 2011;70:1517-8.

23. Cervera R, Serrano R, Pons-Estel GJ, Ceberio-Hualde L, Shoenfeld Y, de Ramón E, et al. Morbidity and mortality in the antiphospholipid syndrome during a 10-year period: a multicentre prospective study of 1000 patients. Ann Rheum Dis 2014; Jan 24 (E-pub ahead of print).

24. Winearls CG, Glassock RJ. Classification of chronic kidney disease in the elderly: pitfalls and errors. Nephron Clin Pract 2011;119 Suppl 1:c2-4.

25. Ruffatti A, Salvan E, Del Ross T, Gerosa M, Andreoli L, Maina A, et al. Treatment strategies and pregnancy outcomes in antiphospholipid syndrome patients with thrombosis and triple antiphospholipid positivity. A European multicentre retrospective study. Thromb Haemost 2014;112:727-35.

J Rheumatol 2015;42:155-7; doi:10.3899/jrheum.140760 